\title{
Protective effect of crocin on food azo dye tartrazine-induced hepatic damage by improving biochemical parameters and oxidative stress biomarkers in rats
}

\author{
Cigdem Velioglu ${ }^{1}$, Mehmet E. Erdemli², Mehmet Gul ${ }^{3}$, Zeynep Erdemli ${ }^{4}$, Emrah Zayman $^{3}$, \\ Harika G. Bag ${ }^{5}$ and Eyup Altinoz ${ }^{6}$ \\ ${ }^{1}$ Department of Chemistry, Faculty of Science, Karabuk University, Karabuk, Turkey \\ ${ }^{2}$ Department of Medical Biochemistry, Faculty of Medicine, Nigde Omer Halisdemir University, Nigde, Turkey \\ ${ }^{3}$ Department of Histology and Embryology, Faculty of Medicine, Inonu University, Malatya, Turkey \\ ${ }^{4}$ Department of Medical Biochemistry, Faculty of Medicine, Inonu University, Malatya, Turkey \\ ${ }^{5}$ Department of Biostatistics, Faculty of Medicine, Inonu University, Malatya, Turkey \\ ${ }^{6}$ Department of Medical Biochemistry, Faculty of Medicine, Karabuk University, Karabuk, Turkey
}

\begin{abstract}
The objective of the present study was to demonstrate the protective effect of crocin on the adverse effects of tartrazine on liver. Crocin is a carotenoid and a strong free radical scavenger. Forty rats were randomly divided into 4 groups $(n=10)$. The first group was the control group (C) and saline solution was administered to this group. The second group (Cr) was administered $50 \mathrm{mg} /$ $\mathrm{kg}$ crocin. The third group $(\mathrm{T})$ was administered $500 \mathrm{mg} / \mathrm{kg}$ tartrazine. The fourth group $(\mathrm{T}+\mathrm{Cr}$ ) was administered the same doses of both crocin and tartrazine as the previous groups for 21 days. It was determined that tartrazine increased liver superoxide dismutase (SOD) activity, malondialdehyde (MDA) and total oxidant status (TOS) levels and catalase (CAT) activity, decreased glutathione (GSH), and total antioxidant status (TAS) levels. Furthermore, tartrazine administration resulted in significant increases in plasma aspartate aminotransferase (AST), alanine aminotransferase (ALT) and alkaline phosphatase (ALP) activities and pathological changes in the liver. When tartrazine administered rats were treated with crocin for 21 days, the biochemical parameters improved, and liver tissues were restored. Thus, it was demonstrated that crocin had protective effects on the adverse effects caused by tartrazine administration.
\end{abstract}

Key words: Tartrazine - Crocin - Oxidative stress - Hepatotoxicity

\section{Introduction}

Tartrazine [trisodium 5-hydroxy-1-(4-sulfonatophenyl)4-[(E)-(4-sulfonatophenyl) diazenyl]-1H-pyrazole-3-carboxylate] is a synthetic monoazo dye that contains pyrazoline ring. Tartrazine (E-102), also known as azo dye since it contains a $-\mathrm{N}=\mathrm{N}$ - group, is an orange-yellow powder dye readily soluble in water and forms golden colored solutions (Deshpande 2002). Tartrazine is frequently used as a food

Correspondence to: Eyup Altinoz, Department of Medical Biochemistry, Faculty of Medicine, Karabuk University, Karabuk, Turkey

E-mail: eyupaltinoz@karabuk.edu.tr additive in aromatic beverages, canned foods, ice creams, confectionery, and in many human drugs and cosmetic products (Mpountoukas et al. 2010; Mahfouz and Al-Shammrani 2013). Used in several countries around the world, tartrazine is the second most commonly used food dye in the United States since 1916. The Food and Drug administration (FDA) established acceptable daily intake (ADI) for tartrazine of 0-7.5 mg/kg/day (Walton et al. 1999).

The maximum daily intake of tartrazine was determined as $5 \mathrm{mg} / \mathrm{kg} /$ day by the FDA.

The primary mechanism in rat, rabbit and human metabolisms of tartrazine taken with foods is the formation of aromatic amines by azo-reductases from intestinal flora. The presence of electron carriers released by bacteria and 
the anaerobic conditions in the colon cause the reduction of tartrazine to two metabolites as sulfanilic acid (an aromatic amine) and aminopyrazolone (Chung et al. 1978; Watabe et al. 1980). It was suggested that the mutagenic, carcinogenic and toxic effects of azo dyes such as tartrazine may be the result of the reductive biotransformation of the azo bond during the metabolism of the dye (Demirkol et al. 2012; Soares et al. 2015). Although intestinal microbial flora plays an important role in tartrazine metabolism, other enzymes in the liver may also break the azo bonds and reduce the nitro groups (Soares et al. 2015). As a result of the reactions both in the intestines and liver, if azo dyes are reduced to fully aromatic amines, these aromatic amines are then oxidized to N-hydroxy derivatives with cytochrome P450 enzymes (Demirkol et al. 2012). These metabolites have the potential to alter the cell cycle through the interphase and in the regenerative hyperplasia process. Therefore, they can have a significant contribution to the development of cancer (Bezerra et al. 2016).

Reactive oxygen species (ROS) occur continuously as a product of aerobic metabolism in living organisms, but they may be over-produced in pathophysiological conditions. Oxidative stress is the degradation of the balance between ROS production and cellular antioxidant defense systems in favor of oxidants (Persson et al. 2014). Detoxification of ROS is one of the prerequisites for aerobic life and there are antioxidant defense systems in living organisms. These defense systems are non-enzymatic, such as reduced glutathione (GSH) and enzymatic systems such as superoxide dismutase (SOD), glutathione peroxidase (GPx), catalase (CAT) (Droge 2002).

Saffron (Crocus sativus L.) is an iridaceous plant indigenous to and planted in Iran, Kashmir (India and Pakistan), Greece, Azerbaijan, China, Morocco, Mexico, Libya, Turkey and Austria. The most important components of the chemical structure of saffron are crocin, crocetine and safranal. Crocin is a carotenoid pigment and has the structure of crocetin di-gentiobiose ester (Bathaie and Mousavi 2010). In recent studies, it has been shown that saffron and its main components (crocin, crocetin and safranal) have several beneficial effects in mice such as hypolipidemic, hypoglycemic, antiatherosclerotic, nephroprotective and antitumor effects (Kianbakht and Hajiaghaee 2011; Bathaie and Mousavi 2013). It is also known that crocin has a strong free radical scavenger and antioxidant properties (Hosseinzadeh et al. 2009b). Recently, phytochemical treatments have been one of the best solutions for overcoming hepatotoxicity through free radical regulation mechanisms (Hassan et al. 2012).

We considered in the present study that the hepatotoxicity induced by tartrazine and its metabolites was a consequence of the increase in ROS. The most important objective of the present study was to provide evidence for the therapeutic potential of crocin in tartrazine-induced liver toxicity.

\section{Material and Methods}

\section{Animals}

The study was conducted with 40 female Wistar albino rats that weighed 225-250 $\mathrm{g}$ and procured from Inonu University, Faculty of Medicine, Experimental Animals Breeding and Research Center (INUTF-DEHUM). The study was approved by the Inonu University experimental animal's ethics committee (2016/A-96). Rats were kept under $21^{\circ} \mathrm{C}$, 55-60\% humidity, 12 hours light (08:00-20:00) : 12 hours dark conditions. Rats were fed ad libitum with standard pellet feed in the study. Drinking water was supplied daily and rat cages were cleaned every day.

\section{Experimental design}

Rats were divided into 4 groups of 10 rats in each group: control (C) group was administered only physiological saline solution; crocin (Cr) group was administered $50 \mathrm{mg} / \mathrm{kg} /$ day crocin (Hosseinzadeh et al. 2009a) (Sigma Aldrich Co., USA, CAS no: 42553-65-1); tartrazine (T) group was administered $500 \mathrm{mg} / \mathrm{kg}$ /day tartrazine (Amin et al. 2010) (Sigma Aldrich Co., USA, CAS no: 1934-21-0); tartrazine+crocin (T+Cr) group was administered $50 \mathrm{mg} / \mathrm{kg} /$ day crocin $+500 \mathrm{mg} /$ $\mathrm{kg} /$ day tartrazine. The applied chemicals were dissolved in saline solution and $1 \mathrm{ml} / \mathrm{kg}$ solution was administered with gavage for 21 days.

\section{Samples}

At the end of the study, laparotomy was conducted on rats under xylazine ketamine anesthesia and blood was drawn from the heart into heparinized tubes. Then, the liver tissues of the decapitated rats were incised and washed with physiological saline to remove the excess blood. One part of the tissue was fixed in $10 \%$ formaldehyde and the other part was stored at $-80^{\circ} \mathrm{C}$ for biochemical analyzes.

\section{Biochemistry}

On the day of the analysis, the tissues were removed from the deepfreeze and weighed. Phosphate buffer was added to obtain $10 \%$ homogenates and the tissues were homogenized for 1-2 minutes in ice at 12,000 rpm (IKA Ultra Turrax T 25 basic, IKA Labotechnik, Staufen, Germany). Homogenates were tested for malondialdehyde (MDA) levels. The supernatants were obtained by centrifuging the remaining homogenates at $600 \times g$ for 30 minutes at $+4^{\circ} \mathrm{C}$. GSH, SOD, CAT, total antioxidant status (TAS), total oxidant status (TOS) and protein levels were studied on the supernatant.

MDA analysis was conducted with the method described by Ohkawa et al. (1979). Tissue homogenate was mixed with 
$1 \% \mathrm{H} 3 \mathrm{PO} 4$ and $0.6 \%$ thiobarbituric acid. The mixture was heated in water bath for 45 minutes and then extracted in nbutanol; n-butanol was used as the blind and tetramethoxypropane was used as the standard. MDA level was measured with a spectrophotometer (T80 UV/VIS Spectrometer, PG Instruments Ltd., Leicestershire, UK) at $535 \mathrm{~nm}$. The results are expressed in $\mathrm{nmol} / \mathrm{g}$ wet tissue.

GSH level was measured based on the method described by Ellman (1959). After 5,5'-dithiobis 2-nitrobenzoic acid (DTNB) (Sigma Aldrich Co., USA, CAS no: 69-78-3) was added in the tubes, a yellow-green color is formed as a result of the reaction between DTNB and the glutathione in the medium. The reduced glutathione amount was determined by measuring the color density at $410 \mathrm{~nm}$ with a spectrophotometer. The results are expressed in $\mathrm{nmol} / \mathrm{g}$ wet tissue.

SOD activity was measured by the method reported by Sun and Oberley (1988). Superoxide radicals are produced by xanthine-xanthine oxidase. The superoxide radical forms a color by reducing NBT (nitro blue tetrazolium) (Sigma Aldrich Co., USA, CAS no: 298-93-9) to blue colored formazan. The absorbance of the produced formazan at $560 \mathrm{~nm}$ is used to calculate the SOD activity. Distilled water was used as the blind. SOD activity was expressed in U/g protein.

CAT activity was measured using the method reported by Aebi et al. (1984). Hydrogen peroxide $\left(\mathrm{H}_{2} \mathrm{O}_{2}\right)$ absorbs ultraviolet radiation; the maximum absorption wavelength is $240 \mathrm{~nm}$. The separation of $\mathrm{H}_{2} \mathrm{O}_{2}$ from water and oxygen by the catalase enzyme in the supernatant leads to a decrease in the absorbance at $240 \mathrm{~nm}$. The reduction observed in the absorption was recorded for 1 minute to measure the enzyme activity. CAT activity was expressed in $\mathrm{K} / \mathrm{g}$ protein.

The tissue homogenate protein content was calculated with the Lowry method (Lowry et al. 1951).

TOS level was measured with the method developed by Erel (2005). It was measured using a total oxidant status kit (Rel Assay Diagnostics, Gaziantep, Turkey). The results are expressed in $\mu \mathrm{mol} \mathrm{H}_{2} \mathrm{O}_{2}$ equiv./l.

The TAS level was studied with the Erel method (Erel 2004). TAS was measured with a Rel Assay brand kit (Rel Assay Diagnostics, Gaziantep, Turkey). Results are expressed in mmol trolox equiv./l.

Plasma aspartate aminotransferase (AST; Architect/ Aeroset Aspartate Aminotransferase Reagent Kit), alanine aminotransferase (ALT; Architect/Aeroset Alanine Aminotransferase Reagent Kit) and alkaline phosphatase (ALP; Architect/Aeroset Alkaline Phosphatase Reagent Kit) levels were determined with the respective kits and the enzymatic colorimetric method in an auto-analyzer (Architect C8000).

\section{Histology}

For the histopathological examination, liver tissue samples were fixed with $10 \%$ formaldehyde containing phosphate buffer in the room temperature for 48 hours. After they were fixed, liver tissue samples were passed through ethanol series with increasing degrees (50, 70, 96 and 99.9\%) for dehydration. They were then passed through xylene series for transparency and molten paraffin series at $62^{\circ} \mathrm{C}$ for infiltration, and then embedded in paraffin blocks. Five-six $\mu \mathrm{m}$ thick sections were prepared with the paraffin blocks using a microtome. Hematoxylin-eosin (H-E) and periodic acid-Schiff (PAS) staining were performed on the sections transferred into slides. The sections were examined with Nikon Eclipse Ni-U light microscope, photographed with a Nikon DS-Fi2 camera and analyzed with a Nikon NISElements Documentation image analysis software (Nikon Corporation, Tokyo, Japan).

Histopathologic deviations (focal necrosis, inflammatory cell infiltration, hydropic degeneration in hepatocytes, vascular and sinusoidal congestion) in liver sections that were stained with $\mathrm{H}$-E were scored between 0 and 3 (0, N/A; 1 , mild-rare; 2 , moderate; 3 , severe-prevalent), so that the maximum total score would be 12 . In sections stained with PAS, intracytoplasmic glycogen content in hepatocytes was scored between 0 and 3 ( 0 , no positive staining; 1 , weak-minimal staining; 2 , moderate staining; 3 , strong-prevalent staining).

\section{Statistical analysis}

Statistical analysis was conducted with SPSS 21 software. Shapiro-Wilk test was used to determine the normal distribution of the data, and Levene test was used to determine the homogeneity of the variances. Data with a normal distribution were summarized with mean and standard deviation values, and data with homogeneous variances were analyzed with one-way analysis of variance and Tukey's honestly significant difference (Tukey HSD) paired comparison method. Since the group variances were not homogeneous, the Welch test and the Games-Howell paired comparison method were used to compare the GSH levels. Median, minimum and maximum values were used to summarize data without normal distribution, and Kruskal-Wallis test and Conover paired comparison method were used for group comparisons. Significance level was accepted as $p<0.05$ in all tests.

\section{Results}

\section{Biochemistry}

Liver tissue oxidant-antioxidant parameters are presented in Table 1 for all groups. Tartrazine administration resulted in a significant increase $(p<0.05)$ in liver MDA levels when compared to $\mathrm{C}(65.8 \%)$ and $\mathrm{Cr}$ groups, while MDA levels in $\mathrm{T}+\mathrm{Cr}$ group decreased significantly $(p<0.05)$ when compared to and $\mathrm{T}$ group. 
Table 1. Liver tissue oxidant-antioxidant parameters of all groups

\begin{tabular}{|c|c|c|c|c|c|c|}
\hline Group & $\begin{array}{c}\text { MDA } \\
\text { (nmol/gwt) }\end{array}$ & $\begin{array}{c}\mathrm{GSH} \\
(\mathrm{nmol} / \mathrm{gwt})\end{array}$ & $\begin{array}{c}\text { SOD } \\
\text { (U/g protein) }\end{array}$ & $\begin{array}{c}\text { CAT } \\
\text { (K/g protein) }\end{array}$ & $\begin{array}{c}\text { TAS } \\
(\mathrm{mmol} / \mathrm{l})\end{array}$ & $\begin{array}{c}\text { TOS } \\
(\mu \mathrm{mol} / \mathrm{l})\end{array}$ \\
\hline $\mathrm{C}$ & $391.80 \pm 31.41^{\#, *, \&}$ & $1191.92 \pm 178.73^{\#, *, \&}$ & $17.42 \pm 1.25^{\#, x, \&}$ & $16.69 \pm 2.09^{\#, *}$ & $1.42 \pm 0.06^{*, \&}$ & $16.93 \pm 2.69^{*, \&}$ \\
\hline $\mathrm{Cr}$ & $244.49 \pm 19.83^{\S, x, \&}$ & $2043.19 \pm 234.01^{\S, *}$ & $10.09 \pm 0.93^{\Im_{,}, \&}$ & $34.50 \pm 2.20^{\S, *, \&}$ & $1.45 \pm 0.06^{\star, \&}$ & $18.07 \pm 2.70^{\star, \&}$ \\
\hline $\mathrm{T}$ & $649.59 \pm 21.36^{\S, \#, \&}$ & $935.35 \pm 43.48^{\S, \#, \&}$ & $26.64 \pm 1.78^{\S, \#, \&}$ & $10.60 \pm 2.43^{\S, \#, \&}$ & $1.06 \pm 0.03^{\S, \#, \&}$ & $31.04 \pm 3.10^{\S, \#, \&}$ \\
\hline $\mathrm{T}+\mathrm{Cr}$ & $563.51 \pm 34.06^{\S, \#, \star}$ & $1764.35 \pm 159.05^{\S, \star}$ & $21.19 \pm 1.57^{\S, \#, *}$ & $14.22 \pm 1.00^{\#, *}$ & $1.21 \pm 0.08^{\S, \#, *}$ & $24.24 \pm 2.11^{\S, \#, *}$ \\
\hline
\end{tabular}

Data are expressed as mean \pm standard deviation $(n=10)$. Superscripts represent the statistically significant difference. ${ }^{\S} p<0.05 v s$. C group; ${ }^{\#} p<0.05$ vs. Cr group; ${ }^{*} p<0.05$ vs. T group; ${ }^{\circledR} p<0.05$ vs. T+Cr group. C, control; Cr, crocin; T, tartrazine; MDA, malondialdehyde; GSH, reduced glutathione; SOD, superoxide dismutase; CAT, catalase; TAS, total antioxidant status; TOS, total oxidant status; gwt, gram wet tissue.

It was observed that GSH levels increased significantly $(p<0.05)$ in the Cr group (71.4\%) when compared to the $\mathrm{C}$ group, while the GSH levels in the T group decreased significantly $(p<0.05)$ when compared to the $C(21.5 \%)$ and Cr groups. Compared to T group, the decreased GSH levels demonstrated a significant increase in the $\mathrm{T}+\mathrm{Cr}$ group $(p<$ 0.05) and even approached Cr group values.

Tartrazine administration resulted in a significant increase in SOD enzyme activity in liver tissue when compared to C (53.0\%) and Cr groups ( $p<0.05)$, while SOD activity significantly decreased in $\mathrm{T}+\mathrm{Cr}$ group when compared to the $\mathrm{T}$ group. Crocin administration resulted in a significant increase $(p<0.05)$ in CAT activity in Cr group when compared to the C group (106.8\%) Conversely, when tartrazine administration led to a significant decrease in liver CAT enzyme activity $(36.5 \%)(p<0.05)$, the same enzyme activity increased significantly $(p<0.05)$ in the $\mathrm{T}+\mathrm{Cr}$ group when compared to the $\mathrm{T}$ group and approached control group levels.

It was determined that tartrazine administration significantly decreased liver TAS levels $(p<0.05)$ when compared to $\mathrm{C}(25.7 \%)$ and $\mathrm{Cr}$ groups, while the decreased TAS levels in the T group significantly increased in $\mathrm{T}+\mathrm{Cr}$ group $(p<0.05)$. Conversely, tartrazine administration led to significant increases $(p<0.05)$ in TOS levels when compared to $\mathrm{C}(83.3 \%)$ and $\mathrm{Cr}$ groups, TOS levels were significantly lower $(p<0.05)$ in the $\mathrm{T}+\mathrm{Cr}$ group when compared to the $\mathrm{T}$ group.
Plasma AST, ALT and ALP values significantly increased $(p<0.05)$ when compared to the $\mathrm{C}$ and $\mathrm{Cr}$ groups after tartrazine administration due to the liver damage, while the same values decreased significantly in the $\mathrm{T}+\mathrm{Cr}$ group $(p<0.05)$ when compared to the T group. Plasma AST, ALT and ALP levels of all groups are presented in Table 2.

\section{Histology}

Hepatocyte cords, sinusoids, central venous and portal areas in the $\mathrm{C}$ (Figure 1A-D) and Cr group (Figure 1E-H) hepatic parenchyma sections after $\mathrm{H}$-E staining were in normal hepatic lobular appearance. Radially organized hepatocyte cords were observed in hepatic lobules, around the central vein. The sinusoid lumen located between the hepatocyte cords reflected clear and normal histological appearance. The hepatocyte cytoplasm was homogeneous and eosinophilic stained in mild to moderate intensity. Hepatocyte nuclei were centrally located with smooth contours and euchromatic structure. Central veins, portal triad connective tissues and vascular structures and bile ducts were open and in normal histological appearance. Kupffer cells were observed at normal density and with normal microscopic structure. Intracytoplasmic glycogen accumulation was prevalent and at intense-medium dense PAS + staining level in PAS-stained sections (Figure 1D and $\mathrm{H}$ ).

Hepatic lobulation was normal in the T group (Figure 2A-D) liver sections stained with H-E. However, hydropic

Table 2. Plasma AST, ALT and ALP levels of all groups

\begin{tabular}{lccc}
\hline Group & AST (min.-max. $)$ & ALT (min.-max.) & ALP (min.-max.) \\
\hline C & $122.0^{*, \&}(92.0-192.0)$ & $82.5^{\star}(47.0-99.0)$ & $152.5^{\star}(140.0-194.0)$ \\
Cr & $108.0^{*}(61.0-144.0)$ & $63,5^{\star}(20.0-98.0)$ & $115.0^{\star}(103.0-142.0)$ \\
$\mathrm{T}$ & $187.0^{\S, \#, \&}(148.0-351.0)$ & $111.5^{\S, \#, \&}(81.0-388.0)$ & $256.0^{\S, \#, \&}(190.0-378.0)$ \\
$\mathrm{T}+\mathrm{Cr}$ & $108.5^{\S, *}(88.0-216.0)$ & $66.0^{\star}(42.0-142.0)$ & $171.5^{\star}(113.0-254.0)$ \\
\hline
\end{tabular}

Data (U/l) are summarized with median, minimum and maximum values $(n=10) .{ }^{\S} p<0.05$ v. C group; ${ }^{\#} p<$ 0.05 vs. Cr group; ${ }^{*} p<0.05 v$ s. T group; ${ }^{\&} p<0.05 v$ s. T+Cr group. AST; aspartate aminotransferase; ALT; alanine aminotransferase; ALP; alkaline phosphatase. For more abbreviations, see Table 1. 
degeneration was prevalent in moderate to severe levels in hepatocytes, and focal necrosis regions and apoptotic bodies in different diameters were identified. Inflammatory cell infiltration in periportal regions and dense congestion in central veins, sinusoids and vascular structures in portal areas were noted. Slightly-minimal PAS + staining observed in PAS-stained sections was considered as a significant reduction in intracytoplasmic glycogen content (Figure 2E).
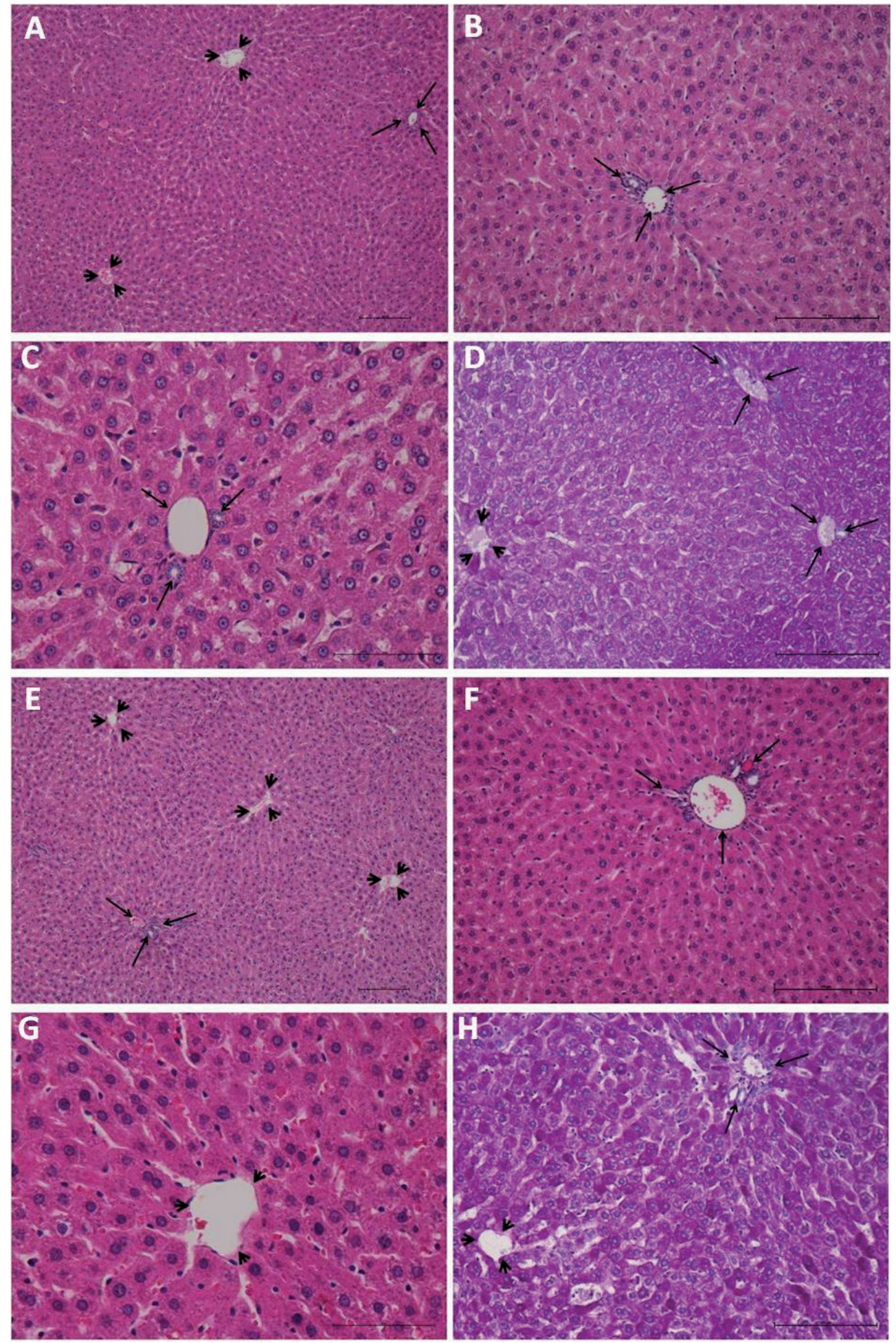

Figure 1. Liver tissues photomicrographs of control group (A-D) and $\mathrm{Cr}$ group (E-H). Magnification for H-E staining: $\times 10(\mathrm{~A}, \mathrm{E})$, $\times 20(\mathrm{~B}, \mathrm{~F}), \times 40(\mathrm{C}, \mathrm{G})$ and for PAS staining: $\times 20(\mathrm{D}, \mathrm{H})$. Central vein (arrowheads); portal triad (arrows); Cr, crocin; H-E, hematoxylineosin; PAS, periodic acidSchiff. 

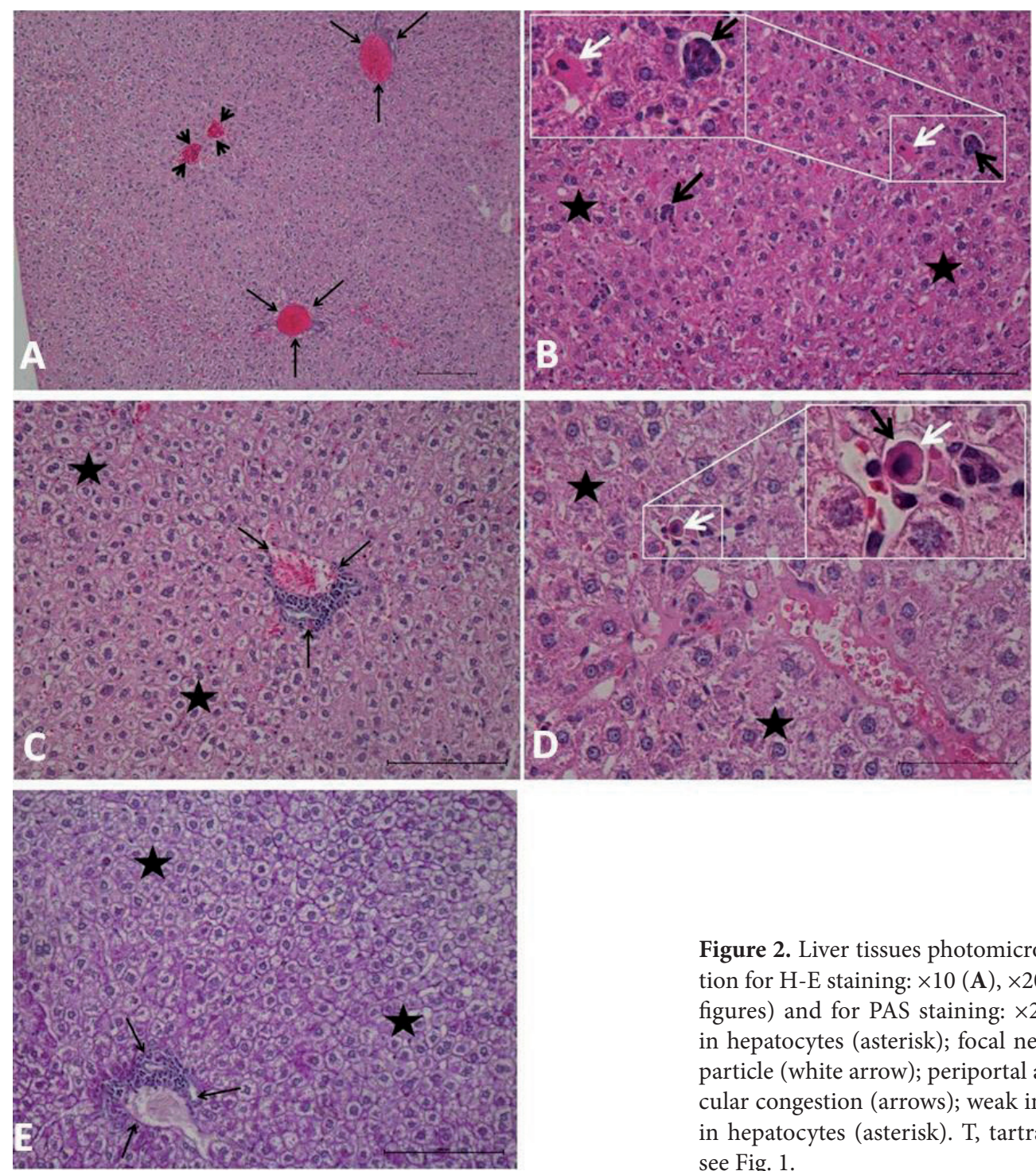

Figure 2. Liver tissues photomicrographs of $\mathrm{T}$ group. Magnification for H-E staining: $\times 10(\mathbf{A}), \times 20(\mathbf{B}, \mathbf{C}), \times 40,(\mathbf{D}), \times 100$ (inside figures) and for PAS staining: $\times 20($ E). Hydropic degeneration in hepatocytes (asterisk); focal necrosis (black arrow); apoptotic particle (white arrow); periportal area cell inflammation and vascular congestion (arrows); weak intracytoplasmic PAS + staining in hepatocytes (asterisk). T, tartrazine. For more abbreviations, see Fig. 1.

Rare focal necrosis areas and hepatocyte groups that exhibited limited regions of mild hydropic degeneration symptoms were determined in the parenchyma of the $\mathrm{H}$-E-stained $\mathrm{T}+\mathrm{Cr}$

Table 3. H-E-stained liver tissue damage scores

\begin{tabular}{lccc}
\hline Group & median & min. & $\max$. \\
\hline $\mathrm{C}$ & $0^{*}, \&$ & 0 & 0 \\
$\mathrm{Cr}$ & $0^{*}, \&$ & 0 & 0 \\
$\mathrm{~T}$ & $5^{\S, \#, \&}$ & 3 & 6 \\
$\mathrm{~T}+\mathrm{Cr}$ & $2^{\S, \#, *}$ & 0 & 3 \\
\hline
\end{tabular}

Data are summarized with median, minimum and maximum values for histological scoring. ${ }^{\S} p<0.05$ vs. C group; ${ }^{\#} p<0.05$ vs. Cr group; ${ }^{*} p<0.05$ vs. T group; ${ }^{\&} p<0.05$ vs. T+Cr group. $\mathrm{H}-\mathrm{E}$, hematoxylin-eosin. For more abbreviations, see Table 1 . group (Figure $3 \mathrm{~A}-\mathrm{C}$ ) liver sections. In some portal regions, minimal inflammatory cell infiltration was observed. Minimal congestion was observed in vascular structures in central veins, sinusoids and vascular structures in the portal triads. However, in general, there was a significant decrease in all damage symptoms, an increase in PAS + staining prevalence and intensity when compared to the T group (Figure 3D). HE-stained liver tissue damage scores are presented in Table 3 and PAS-stained liver tissue scores are presented in Table 4.

\section{Discussion}

The aim of this study was to establish liver damage caused by a food dye, tartrazin, and to determine the protective ef- 
fect of crocin against this disorder by biochemical tests and histological examinations.

The synthetic food dyes are widely used in the food industry since they are relatively durable and inexpensive (Nayak and Nath 2010). The first catabolic step in the reduction of azo dyes is the reduction of the azo bond to produce aromatic amines accompanied by a reduction in visible light absorbance and subsequent degradation of the dye color (Soares et al. 2015). The aromatic amines, some of which are known as carcinogens, were found in urine of dye manufacturing workers and experimental animals after administration of azo dyes (Cerniglia et al. 1986). Furthermore, Siraki et al. (2002) found that incubation of hepatocytes with aromatic amines caused a reduction in mitochondrial membrane potential before cytotoxicity was observed. Tartrazine causes changes in the biochemical profiles of kidney and liver tissues, and concurrently, leads to a risk at higher doses and results in oxidative stress in the tissues via free radical formation (Himri et al. 2011). The antioxidant system is involved in the free radical-mediated tissue or cellular damage defense system (Mourad and Noor 2011). In the present study, daily oral administration of tartrazine to rats for twenty-one days resulted in suppression of the antioxidant system. It was found that tartrazine administration significantly increased the levels of SOD, the primary free radical scavenging antioxidant enzyme that detoxifies superoxide $\left(\mathrm{O}^{2-}\right)$ (Arjuman et al. 2007). Tartrazine and its
Table 4. PAS-stained liver tissue scores

\begin{tabular}{lccc}
\hline Group & median & min. & $\max$. \\
\hline $\mathrm{C}$ & $3^{*}$ & 2 & 3 \\
$\mathrm{Cr}$ & $3^{*}$ & 2 & 3 \\
$\mathrm{~T}$ & $1^{\S, \#, \&}$ & 1 & 2 \\
$\mathrm{~T}+\mathrm{Cr}$ & $2^{*}$ & 1 & 3 \\
\hline
\end{tabular}

Data are summarized with median, minimum and maximum values for histological scoring. ${ }^{\S} p<0.05$ vs. C group; ${ }^{\#} p<0.05 v s$. Cr group; ${ }^{\star} p<0.05$ vs. T group; ${ }^{\&} p<0.05 v s$. T+Cr group. PAS, periodic acid-Schiff. For more abbreviations, see Table 1 .

metabolites may cause the formation of ROS in the tissue and the increased ROS leads to an increase in cellular SOD activity and $\mathrm{H}_{2} \mathrm{O}_{2}$ levels. However, due to the serious decreases observed in CAT activity after tartrazine administration, increased cellular $\mathrm{H}_{2} \mathrm{O}_{2}$ could not be sufficiently detoxified, resulting in oxidative stress in the liver. Recent studies on the administration of food dyes demonstrated that food dyes increase the production of ROS, leading to oxidative stress (Himri et al. 2011; Visweswaran and Krishnamoorthy 2012). The increase in ROS production such as superoxide anion, hydroxyl radical and $\mathrm{H}_{2} \mathrm{O}_{2}$ in an organism is correlated with the decrease in antioxidant defense mechanism of cell including SOD, CAT and GSH to prevent the cell death by these toxic radicals and leads to oxidative stress (El-Tohamy
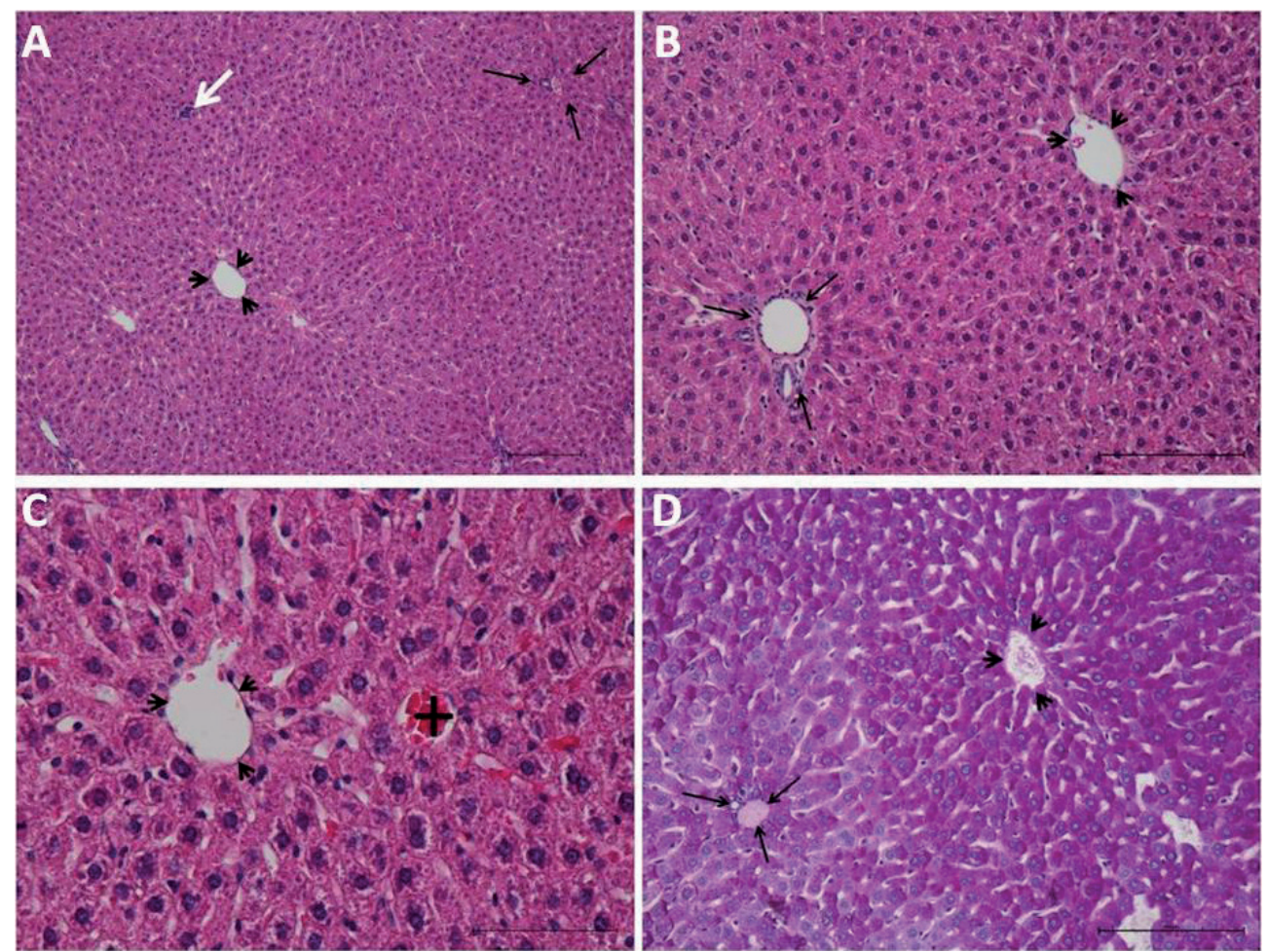

Figure 3. Liver tissues photomicrographs of $\mathrm{T}+\mathrm{Cr}$ group. Magnification for H-E staining: $\times 10(\mathbf{A}), \times 20$ (B), $\times 40($ C $)$ and for PAS staining: $\times 20($ D $)$. Central vein (arrowheads); portal triad (black arrow); focal necrosis (white arrow); congestion (plus). For more abbreviations, see Fig. 1. 
2012). Oxidative stress is caused by the production of free radicals and the destruction of the oxidant-antioxidant balance, where the free radicals are scavenged by the antioxidant system (Friederich et al. 2009; Kaneto et al. 2010). On the other hand the MDA levels were increased as an indicator of lipid peroxidation by the ROS effect on the lipids of the cellular membrane. Interestingly, crocin treatment resulted in significant improvement in SOD and CAT activities when compared to the tartrazine group, and even resulted in close to control levels CAT activity. Gao et al. (2011) reported that tartrazine administration resulted in a decrease in CAT, SOD and glutathione reductase (GR) antioxidant enzyme activities. The findings of the present study were consistent with recent reports that showed reduced antioxidant enzyme activities in the liver (Amin et al. 2010) and brain (Gao et al. 2011) tissues of tartrazine administered rats. However, in the present study, SOD activity increased after tartrazine treatment, while Amin et al. (2010) reported that SOD activity decreased after administration of tartrazine to rats.

Similar to previous studies (Zheng et al. 2007), the present study revealed that crocin can protect tissues via the mechanisms that regulate the antioxidant defense system.

GSH is a non-enzymatic antioxidant that plays an active role in endogenous antioxidant defense, neutralizing free radicals and reducing hydrogen peroxide levels (Meister and Anderson 1983). In the present study, we observed that the GSH level significantly decreased with tartrazine administration. GSH depletion caused oxidative damage in the liver due to the imbalance in the oxidant/antioxidant system. Conversely, it was observed that the tartrazine administration significantly increased MDA levels. On the other hand, it was found that when treated with crocin, there was a statistically significant decrease in MDA levels accompanied by a significant increase in GSH levels when compared to the tartrazine group. Amin et al. (2010) demonstrated that GSH, SOD, and CAT were significantly decreased and MDA increased in liver tissue homogenates in rats consumed high dose tartrazine $(500 \mathrm{mg} / \mathrm{kg}$ ) and both doses of carmoisine (8 and $100 \mathrm{mg} / \mathrm{kg}$ ) for 30 days. Also, Erdemli et al. (2017) reported that there was a significant increase in MDA, TOS, SOD, CAT, BUN (blood urea nitrogen) and creatinine levels, while a significant decrease in GSH and TAS levels in tartrazine administered rat kidney tissues. Although our study is compatible with the studies of Amin et al., it differs with the studies of Erdemli et al. The reason for this difference is that in our study, excessive ROS production and degradation of the antioxidant enzyme activities may be caused due to high exposure of liver to tartrazine and its metabolites because of tartrazine is primarily transported to the liver after the digestive system and is metabolized by cytochrome P450 enzymes. Previous studies demonstrated that crocin increased intracellular GSH levels, and thus preventing necrosis in hypoxic PC12 cells (Ochiai et al. 2004). Furthermore, Ochiai et al. (2007) reported that saffron extract and crocin reduced free radical-induced lipid peroxidation and MDA levels due to their antioxidant properties. Hosseinzadeh et al. (2005, 2009a) reported that saffron extract and crocin significantly reduced ischemia reperfusion-induced oxidative stress in rat tissues.

Biological membranes are particularly prone to ROS attack, and toxic radicals cause peroxidation of unsaturated fatty acids in membranes. This leads to reduced membrane fluidity and degradation of membrane integrity and function, leading to severe pathological changes (Halliwell 1987). Increased ROS or free radical production may lead to autoxidation in hepatic cells, resulting in explicit liver lesions (Suzuki et al. 2001). In a study, administration of high doses of tartrazine, a food coloring agent, to rats leads to elevated plasma enzyme activities (AST, ALT and ALP), increased hepatocyte permeability, damages and injuries (Stryer 1995). Since ALT enzymes are normally localized in the cytoplasm, AST is mainly localized in organelles such as the mitochondria (Rajagopal et al. 2003). Increased AST and ALT levels suggested that food azo dyes administered to rats led to hepatic cellular and mitochondrial membrane damage. Mekkawy et al. (1998) found that serum ALT, AST and ALP levels were significantly increased when they administered tartrazine and carmoisine synthetic dyes. The present study findings were consistent with those of the previous studies, and tartrazine administration caused liver damage and an increase in plasma AST, ALT and ALP levels. Khayyat et al. (2017) observed an increase in plasma AST, ALP, and ALT after tartrazine administration. In a different study, tartrazine-administered rats exhibited elevated AST, ALP and ALT levels (Al-Seeni et al. 2017). In our study, when we applied tartrazine and crocin together, we observed a significant decrease in plasma levels of these liver enzymes compared to tartrazine group. The results of the present study were consistent with previous studies where the AST, ALT, and ALP levels, elevated by hepatotoxicity induced by morphine (Salahshoor 2016), amiodarone (Riaz et al. 2016), patulin (Boussabbeh et al. 2016) and acetaminophen (Omidi et al. 2014) administration in animal models, significantly decreased with crocin treatment.

Consistent with our biochemical findings, histological results demonstrated that the use of tartrazine led to moderate to severe degeneration in the liver, focal necrosis and intense congestion and inflammatory cell infiltration. It was demonstrated that the tartrazine-induced damage was minimized when treated with crocin. In similar histopathologic studies, brown pigment accumulation in kidney and hepatic portal tracts and Van Kupffer cells induced by the coloring carmoisine was demonstrated (Aboel-Zahab et al. 1997).

In conclusion, the present study demonstrated that the tartrazine-induced rat liver damage was due to oxidative stress, and this damage was eliminated by crocin treatment 
based on the biochemical and histological findings. Crocin has antioxidant properties due to its ROS scavenging effect. Thus, elevated plasma ALT, AST and ALP levels and liver MDA, TOS and SOD levels after tartrazine administration decreased with crocin treatment, while decreased GSH and TAS levels and CAT activity increased after the treatment. Thus, it is required to raise consumer awareness on the adverse effects of these azo dyes, and to indicate the type and concentration of each ingredient included in food products.

Based on results we suggest to "rise of consumer awareness". However, dose used in this study $500 \mathrm{mg} / \mathrm{kg} /$ day is 100 higher than ADI determined as $5 \mathrm{mg} / \mathrm{kg} /$ day by FDA.

Acknowledgement. The present study was sponsored by Karabuk University scientific research fund (KBU-BAP-16/2YL-093).

Conflict of interest. The authors declared no conflict of interest.

\section{References}

Aboel-Zahab H, El-Khyat Z, Sidhom G, Awadallah R, Abdel-Al W, Mahdy K (1997): Physiological effects of some synthetic food colouring additives on rats. Boll. Chim. Farm. 136, 615-627

Aebi H (1984): Catalase in vitro. Methods in Enzymology. pp. 121-126, Elsevier, Cambridge

Al-Seeni MN, El Rabey HA, Al-Hamed AM, Zamazami MA (2018): Nigella sativa oil protects against tartrazine toxicity in male rats. Toxicol. Rep. 5, 146-155 https://doi.org/10.1016/j.toxrep.2017.12.022

Amin, K, Hameid II HA, Elsttar AA (2010): Effect of food azo dyes tartrazine and carmoisine on biochemical parameters related to renal, hepatic function and oxidative stress biomarkers in young male rats. Food Chem. Toxicol. 48, 2994-2999 https://doi.org/10.1016/j.fct.2010.07.039

Arjuman A, Nair V, Gopalakrishna H, Nandini M (2007): Evaluation of the antioxidant potential of NR-ANX-C (a polyherbal formulation) and its individual constituents in reversing haloperidol-induced catalepsy in mice. Indian J. Pharmacol. 39, 151-154 https://doi.org/10.4103/0253-7613.33435

Bathaie SZ, Mousavi SZ (2010): New applications and mechanisms of action of saffron and its important ingredients. Crit. Rev Food. Sci. Nutr. 50, 761-786 https://doi.org/10.1080/10408390902773003

Bezerra MdS, Malaquias GdS., Castro E Sousa JMd, Peron AP (2016): Cytotoxic and genotoxic potential of powdered juices. Food Sci.Tech. (Campinas) 36, 49-55

Boussabbeh M, Salem IB, Belguesmi F, Neffati F, Najjar MF, AbidEssefi S, Bacha H (2016): Crocin protects the liver and kidney from patulin-induced apoptosis in vivo. Environ. Sci. Pollut. R 23, 9799-9808 https://doi.org/10.1007/s11356-016-6195-2

Cerniglia CE, Zhuo Z, Manning BW, Federle TW, Heflich RH (1986): Mutagenic activation of the benzidine-based dye
Direct Black 38 by human intestinal microflora. Mutat. Res. Lett. 175, 11-16 https://doi.org/10.1016/0165-7992(86)90138-7

Chung K-T, Fulk GE, Egan M (1978): Reduction of azo dyes by intestinal anaerobes. Appl. Environ. Microb. 35, 558-562

Demirkol O, Zhang X, Ercal N (2012): Oxidative effects of Tartrazine (CAS No. 1934-21-0) and New Coccin (CAS No. 2611-827) azo dyes on CHO cells. J. Verbrauch. Lebensm. 7, 229-236 https://doi.org/10.1007/s00003-012-0782-z

Deshpande S (2002): Handbook of Food Toxicology. CRC Press, New York https://doi.org/10.1201/9780203908969

Droge W (2002): Free radicals in the physiological control of cell function. Physiol. Rev. 82, 47-95 https://doi.org/10.1152/physrev.00018.2001

Ellman GL (1959): Tissue sulfhydryl groups. Arch. Biochem. Biophys. 82, 70-77 https://doi.org/10.1016/0003-9861(59)90090-6

El-Tohamy MM (2012): The mechanisms by which oxidative stress and free radical damage produces male infertility. Life Sci. 9, 674-688

Erdemli ME, Gul M, Altinoz E, Zayman E, Aksungur Z, Bag HG (2017): The protective role of crocin in tartrazine induced nephrotoxicity in Wistar rats. Biomed. Pharmacother. 96, 930-935 https://doi.org/10.1016/j.biopha.2017.11.150

Erel O (2004): A novel automated direct measurement method for total antioxidant capacity using a new generation, more stable ABTS radical cation. Clin. Biochem. 37, 277-285 https://doi.org/10.1016/j.clinbiochem.2003.11.015

Erel O (2005): A new automated colorimetric method for measuring total oxidant status. Clin. Biochem. 38, 1103-1111 https://doi.org/10.1016/j.clinbiochem.2005.08.008

Friederich M, Hansell P, Palm F (2009): Diabetes, oxidative stress, nitric oxide and mitochondria function. Curr. Diabetes Rev. $5,120-144$ https://doi.org/10.2174/157339909788166800

Gao Y, Li C, Yin H, An X, Jin H (2011): Effect of food azo dye tartrazine on learning and memory functions in mice and rats, and the possible mechanisms involved. J. Food Sci. 76, 125-129 https://doi.org/10.1111/j.1750-3841.2011.02267.x

Hassan MH, Edfawy M, Mansour A, Hamed A-A (2012): Antioxidant and antiapoptotic effects of capsaicin against carbon tetrachloride-induced hepatotoxicity in rats. Toxicol. Ind. Health 28, 428-438 https://doi.org/10.1177/0748233711413801

Himri I, Bellahcen S, Souna F, Belmekki F, Aziz M, Bnouham M, Zoheir J, Berkia Z, Mekhfi H, Saalaoui E (2011): A 90-day oral toxicity study of tartrazine, a synthetic food dye, in wistar rats. Int. J. Pharm. Pharm. Sci. 3, 159-169

Hosseinzadeh H, Modaghegh MH, Saffari Z (2009a): Crocus sativus L. (Saffron) extract and its active constituents (crocin and safranal) on ischemia-reperfusion in rat skeletal muscle. Evid. Based Compl. Alt. 6, 343-350 https://doi.org/10.1093/ecam/nem125

Hosseinzadeh H, Shamsaie F, Mehri S (2009b): Antioxidant activity of aqueous and ethanolic extracts of Crocus sativus L. stigma and its bioactive constituents, crocin and safranal. Phcog. Mag. $5,419-424$ 
Hosseinzadeh H, Nassiri-Asl M (2013): Avicenna's (Ibn Sina) the Canon of Medicine and saffron (Crocus sativus): A Review. Phytother. Res. 27, 475-483 https://doi.org/10.1002/ptr.4784

Kaneto H, Katakami N, Matsuhisa M, Matsuoka TA (2010). Role of reactive oxygen species in the progression of type 2 diabetes and atherosclerosis. Mediat. Inflamm. 2010, 453892 https://doi.org/10.1155/2010/453892

Khayyat L, Essawy A, Sorour J, Soffar A (2017). Tartrazine induces structural and functional aberrations and genotoxic effects in vivo. Peer J. 5, e3041 https://doi.org/10.7717/peerj.3041

Kianbakht S, Hajiaghaee R (2011): Anti-hyperglycemic effects of saffron and its active constituents, crocin and safranal, in alloxan-induced diabetic rats. J. Med. Plants 3, 82-89

Lowry OH, Rosebrough NJ, Farr AL, Randall RJ (1951): Protein measurement with the Folin phenol reagent. J. Biol. Chem. 193, 265-275

Mahfouz H, Al-Shammrani S (2013): Protective action of vitamin $\mathrm{C}$ against mutagenic effects of synthetic food color tartrazine. Afr. J. Pharm. Pharmaco. 7, 2474-2483 https://doi.org/10.5897/AJPP2013.3561

Meister A, Anderson ME (1983): Glutathione. Annu Rev. Biochem. 52, 711-760 https://doi.org/10.1146/annurev.bi.52.070183.003431

Mekkawy H, Ali M, El-Zawahry A (1998): Toxic effect of synthetic and natural food dyes on renal and hepatic functions in rats. Toxicol. Lett. 95, 155 https://doi.org/10.1016/S0378-4274(98)80621-8

Mourad IM, Noor NA (2011): Aspartame (a widely used artificial sweetener) and oxidative stress in the rat cerebral cortex. Int. J. Pharm. Biomed. Sci. 2, 4-10

Mpountoukas P, Pantazaki A, Kostareli E, Christodoulou P, Kareli D, Poliliou S, Mourelatos C, Lambropoulou V, Lialiaris T (2010): Cytogenetic evaluation and DNA interaction studies of the food colorants amaranth, erythrosine and tartrazine. Food Chem. Toxicol. 48, 2934-2944 https://doi.org/10.1016/j.fct.2010.07.030

Nayak H, Nath KG (2010): Dietary intake of synthetic colours by school children. Karnataka J. Agric. 20, 819-820

Ochiai T, Ohno S, Soeda S, Tanaka H, Shoyama Y, Shimeno H (2004): Crocin prevents the death of rat pheochromyctoma (PC-12) cells by its antioxidant effects stronger than those of a-tocopherol. Neurosci. Lett. 362, 61-64 https://doi.org/10.1016/j.neulet.2004.02.067

Ochiai T, Shimeno H, Mishima K-i, Iwasaki K, Fujiwara M, Tanaka H, Shoyama Y, Toda A, Eyanagi R, Soeda S (2007): Protective effects of carotenoids from saffron on neuronal injury in vitro and in vivo. Biochim. Biophys. Acta 1770, 578-584 https://doi.org/10.1016/j.bbagen.2006.11.012

Ohkawa H, Ohishi N, Yagi K (1979): Assay for lipid peroxides in animal tissues by thiobarbituric acid reaction. Anal. Biochem. 95, 351-358 https://doi.org/10.1016/0003-2697(79)90738-3
Omidi A, Riahinia N, Torbati MBM, Behdani,M-A (2014): Hepatoprotective effect of Crocus sativus (saffron) petals extract against acetaminophen toxicity in male Wistar rats. Avicenna J. Phytomed. 4, 330-336

Persson T, Popescu BO, Cedazo-Minguez A (2014): Oxidative stress in Alzheimer's disease: why did antioxidant therapy fail? Oxid. Med. Cell. Longev. 2014, 427318 https://doi.org/10.1155/2014/427318

Rajagopal SK, Manickam P, Periyasamy V, Namasivayam N (2003): Activity of Cassia auriculata leaf extract in rats with alcoholic liver injury. J. Nutr. Biochem. 14, 452-458 https://doi.org/10.1016/S0955-2863(03)00053-6

Riaz H, Saleem N, Ahmad M, Mehmood Y, Raza SA, Khan S, Anwar R, Kamran SH (2016): Hepatoprotective effect of Crocus sativus on amiodarone-induced liver toxicity. Br. J. Pharm. Res. 12, 1-11

Salahshoor MR (2016): Protective effect of crocin on liver toxicity induced by morphine. Res. Pharm. Sci. 11, 120-129

Siraki AG, Chan TS, Galati G, Teng S O’Brien PJ (2002): Noxidation of aromatic amines by intracellular oxidases. Drug Metab. Rev. 34, 549-564 https://doi.org/10.1081/DMR-120005657

Soares BM, Araújo TMT, Ramos JAB, Pinto LC, Khayat BM, Bahia MDO, Montenegro RC, Burbano RMR, Khayat AS (2015): Effects on DNA repair in human lymphocytes exposed to the food dye tartrazine yellow. Anticancer Res. 35, 1465-1474

Stryer L (1995): Principles of Biochemistry. WH Freeman and Company, New York

Sun Y, Oberley LW, Li Y (1988): A simple method for clinical assay of superoxide dismutase. Clin.Chem. 34, 497-500

Suzuki Y, Ishihara M, Segami T, Ito M (2001): Anti-ulcer effects of antioxidants, quercetin, $\alpha$-tocopherol, nifedipine and tetracycline in rats. Jpn. J. Pharmacol. 78, 435-441 https://doi.org/10.1254/jjp.78.435

Visweswaran B, Krishnamoorthy G (2012): Oxidative stress by tartrazine in the testis of Wistar rats. Journal of Pharmacy and Biological Sciences 2, 44-49 https://doi.org/10.9790/3008-0234447

WaltonK, Walker R, Van de Sandt JJM, Castell JV, Knapp AGAA, Kozianowski G, Schilter B (1999): The application of in vitro data in the derivation of the acceptable daily intake of food additives. Food Chem. Toxicol. 37, 1175-1197 https://doi.org/10.1016/S0278-6915(99)00107-6

Watabe T, Ozawa N, Kobayashi F, Kurata H (1980): Reduction of sulphonated water-soluble azo dyes by micro-organisms from human faeces. Food Cosmet. Toxicol. 18, 349-352 https://doi.org/10.1016/0015-6264(80)90187-X

Zheng Y-Q, Liu J-X, Wang J-N, Xu L (2007): Effects of crocin on reperfusion-induced oxidative/nitrative injury to cerebral microvessels after global cerebral ischemia. Brain Res. 1138, 86-94 https://doi.org/10.1016/j.brainres.2006.12.064

Received: June 25, 2018

Final version accepted: October 3, 2018

First published online: January 18, 2019 